\title{
The definition of the thermodynamic entropy in statistical mechanics
}

\author{
Robert H. Swendsen* \\ The Department of Physics, Carnegie Mellon University, Pittsburgh, PA 15213, USA
}

(Dated: August 18, 2016)

\begin{abstract}
A definition of the thermodynamic entropy based on the time-dependent probability distribution of the macroscopic variables is developed. When a constraint in a composite system is released, the probability distribution for the new equilibrium values goes to a narrow peak. Defining the entropy by the logarithm of the probability distribution automatically makes it a maximum at the equilibrium values, so it satisfies the Second Law. It is also satisfies the postulates of thermodynamics. Objections to this definition by Dieks and Peters are discussed and resolved.
\end{abstract}

Keywords: Entropy; Thermodynamics; Statistical mechanics; Irreversibility; Second law of thermodynamics

\section{INTRODUCTION}

Thermodynamics is an extremely successful phenomenological theory of macroscopic experiments. The entropy plays a central role in this theory because it is a unique function for each system that determines all thermodynamic information. The calculation of the form of the entropy lies in the microscopic description given by statistical mechanics. In this paper, I present a simple derivation of the entropy using reasonable assumptions about the probability distributions of macroscopic variables and approximations based on the large number of particles in macroscopic systems.

The basic task of thermodynamics is the prediction of the values of the macroscopic variables after the release of one or more constraints and the subsequent relaxation to a new equilibrium. This appears in the key thermodynamic postulate that is a particular form of the second law. [1] [3].

The values assumed by the extensive parameters of an isolated composite system in the absence of an internal constraint are those that maximize the entropy over the set of all constrained macroscopic states [3].

I will show that the solution to this problem in statistical mechanics leads to a function that satisfies this postulate, as well as satisfying the rest of the postulates of thermodynamics. Since these postulates are sufficient to generate all of thermodynamics, and since the thermodynamic entropy is unique [4], this function can be identified as the entropy.

I have presented other derivations in the past that are equivalent, though perhaps not as direct $[5-10]$. They have been criticized by Dieks [11, 12] and Peters 13, 14], whose arguments will be discussed in Sections $\mathrm{V}$ and VI

* swendsen@cmu.edu

\section{THE PREDICTION OF EQUILIBRIUM} VALUES FROM STATISTICAL MECHANICS

Thermodynamics is a description of the properties of systems containing many particles (macroscopic systems), for which the fluctuations can be ignored because they are smaller than the experimental resolution. The basic problem of thermodynamics is to predict the equilibrium values of the extensive variables after the release of a constraint in a composite system. I will first consider this as a problem is statistical mechanics, without using any thermodynamic concepts.

Consider a composite system of $M \geq 2$ subsystems, with a total energy $E_{T}$, volume $V_{T}$, and particle number $N_{T}$ [15]. Denote the total phase space for this composite system (in three dimensions) by $\{p, q\}$, where $p$ is the $3 N_{T^{-}}$dimensional momentum space, and $q$ is the $3 N_{T^{-}}$ dimensional configuration space. Define the probability distribution in the phase space of the composite system as $\phi_{T}(\{p, q\}, t)$, where $t$ is the time. I'll assume that the composite system is initially in equilibrium at time $t=0$, and that the initial conditions are given by setting $\phi_{T}$ equal to a constant, subject to all information available about the system at that time.

Assume that interactions between subsystems are sufficiently short-ranged that they may be neglected[16]. Then, we can write the total Hamiltonian as a sum of contributions from each system.

$$
H_{T}=\sum_{j=1}^{M} H_{j}\left(E_{j}, V_{j}, N_{j}\right)
$$

The energy, volume, and particle number of subsystem $j$ are denoted as $E_{j}, V_{j}$, and $N_{j}$, subject to the conditions on the sums.

$$
\sum_{j=1}^{M} E_{j}=E_{T} ; \sum_{j=1}^{M} V_{j}=V_{T} ; \sum_{j=1}^{M} N_{j}=N_{T}
$$

In keeping with the idea that we are describing macroscopic experiments, assume that no measurements are made that might identify individual particles, whether or not they are formally indistinguishable [17]. This means that there are $N_{T}$ ! $\left(\prod_{j=1}^{M} N_{j} !\right)$ different permutations 
for assigning particles to subsystems, and all permutations may be regarded as equally probable. The probability distribution in the phase space of the composite system is given by

$$
\begin{aligned}
\phi_{T}(\{p, q\}, t=0)= & \frac{1}{\Omega_{T}}\left(\frac{N_{T} !}{\prod_{j=1}^{M} N_{j} !}\right) \\
& \times \prod_{k=1}^{M} \delta\left(E_{k}-H_{k}\left(\left\{p_{k}, q_{k}\right\}\right)\right),
\end{aligned}
$$

where $\left\{p_{k}, q_{k}\right\}$ is the phase space for the particles in subsystem $k$, and $\Omega_{T}$ is a normalization factor. The constraint that the $N_{k}$ particles in subsystem $k$ are restricted to a volume $V_{k}$ is left implicit in Eq. (3).

The probability distribution for the macroscopic observables can then be written as

$$
\begin{aligned}
W\left(\left\{E_{j}, V_{j}, N_{j}\right\}\right)= & \frac{N_{T} !}{\Omega_{T}}\left(\frac{1}{\prod_{j} N_{j} !}\right) \\
& \times \int d p \int d q \prod_{j=1}^{M} \delta\left(E_{j}-H_{j}\right),
\end{aligned}
$$

or

$$
W\left(\left\{E_{j}, V_{j}, N_{j}\right\}\right)=\frac{\prod_{j=1}^{M} \Omega_{j}\left(E_{j}, V_{j}, N_{j}\right)}{\Omega_{T} / N_{T} ! h^{3 N_{T}}},
$$

where

$$
\Omega_{j}=\frac{1}{h^{3 N_{j}} N_{j} !} \int_{-\infty}^{\infty} d p_{j} \int_{V_{j}} d q_{j} \delta\left(E_{j}-H_{j}\right) .
$$

The factor of $1 / h^{3 N_{j}}$, where $h$ is Planck's constant, is not necessary for classical mechanics. It has been included to ensure that the final answer agrees with the classical limit from quantum statistical mechanics [3] .

There is no requirement that the Hamiltonians $H_{j}$ are the same, so there is also no requirement that the individual $\Omega_{j}$ 's have the same functional form. Long-range interactions within a system are allowed.

If one or more constraints are now released, the probability $\phi_{T}(\{p, q\}, t)$ will become time dependent. After sufficient time has passed, the probability distribution will have spread throughout the available phase space, although it will still be non-uniform on the finest scale due to Liouville's theorem. The probability distribution for the macroscopic variables will again be given by $W\left(\left\{E_{j}, V_{j}, N_{j}\right\}\right)$, but now without the constraints on the variables that have been released [18]. The functional dependence of $W$ on the variables $\left\{E_{j}, V_{j}, N_{j}\right\}$ does not change when a constraint is released.

An important advantage of working with the probability distributions for macroscopic observables is that they converge to the equilibrium probability distributions at the end of an irreversible process [18]. Although it is not necessary, the introduction of coarse graining [19] or the modification of the microscopic probability distribution by invoking typicality [20, 21] leads to the same results.
Usually, $W$ is a very narrow function of the released variables. The main exception is the case of a first-order phase transition, in which it can be a very broad function of the relevant variable 16]. This situation is discussed in Ref. [16], and I will ignore it for the present discussion.

The location of the narrow peak in $W$ as a function of the variable describing a released constraint gives the final equilibrium value of that variable at the end of the irreversible process. For example, if subsystems 1 and 2 are brought in thermal contact so that energy transfer is possible, the final value of $E_{1}$ would be given by the location of the maximum of $W$ to within thermal fluctuations. This characterizes the equilibrium values as the mode of the probability distribution, not the mean. The difference between the mean and the mode is of the order of $1 / N$, which is very small and far less than the assumed experimental accuracy. Indeed, it is not even measurable for macroscopic systems [22].

When subsystems are separated, the probability $W$ remains unchanged. The constraint is restored, and the variable that was being exchanged keeps its value, which is known to within the very small fluctuations.

The normalization constant, $\Omega_{T}$, is dependent on exactly which constraints might be released, but the other factors are not. Since the only property of the function $W\left(\left\{E_{j}, V_{j}, N_{j}\right\}\right)$ that is needed is that it has a very narrow peak at the equilibrium value(s) after the release of constraint(s), the value of $\Omega_{T}$ does not affect the argument.

Now that the probability distribution for the equilibrium variables has been determined, we can turn to the definition of entropy.

\section{THE DEFINITION OF THE THERMODYNAMIC ENTROPY}

Following Boltzmann [6, 23, 24], the thermodynamic entropy may be identified as the logarithm of the probability distribution $W$, plus an arbitrary constant.

$$
S_{T}\left(\left\{E_{j}, V_{j}, N_{j}\right\}\right)=k_{B} \ln W+X
$$

Since the probability is a maximum at equilibrium, the entropy is also with this definition. Although Boltzmann considered a dimensionless entropy and never used the "Boltzmann constant," $k_{B}$, which was introduced by Planck 25, 26], I have included a factor of $k_{B}$ to be consistent with physical units.

Combining Eqs. (5), (6) and (7), the total entropy can be written as a sum of $M$ terms, each of which depends only on the properties of a single subsystem, plus a constant.

$$
S_{T}=\sum_{j=1}^{M} S_{j}\left(E_{j}, V_{j}, N_{j}\right)-k_{B} \ln \left[\frac{\Omega_{T}}{N_{T} ! h^{3 N_{T}}}\right]+X
$$

The entropy of the $j$-th subsystem in Eq. (8) is given by

$$
S_{j}\left(E_{j}, V_{j}, N_{j}\right)=k_{B} \ln \Omega_{j}\left(E_{j}, V_{j}, N_{j}\right),
$$


or

$$
S_{j}=k_{B} \ln \left[\frac{1}{h^{3 N_{j}} N_{j} !} \int_{-\infty}^{\infty} d p_{j} \int_{V_{j}} d q_{j} \delta\left(E_{j}-H_{j}\right)\right] .
$$

The entropy of subsystem $j$ contains the factor $1 / N_{j}$ !, which arises from the multinomial factor in Eq. (3). It would be possible to add an arbitrary constant $X_{j}$ to $S_{j}$ in Eq. (10), but I have chosen to set $X_{j}=0$ for all $j$, which is the usual convention [9].

$S_{j}$ only depends on the properties of system $j$, which means that the total entropy is separable. This is just the usual thermodynamic property of additivity, but viewed from the perspective of dividing up a composite system, rather than assembling one.

Since $\Omega_{T}$ has been defined to be a normalization constant, if all chosen constraints are released, the value of $S_{T}$ after the composite system has returned to equilibrium is given entirely by the additive constant (neglecting terms of the order of the logarithm of the particle numbers).

$$
S_{T}(\text { after release }) \rightarrow X
$$

This will be true regardless of which constraints have been chosen to determine $\Omega_{T}$, as long as all of those constraints are released.

A convenient choice of $X$ is $k_{B} \ln \left[\Omega_{T} / N_{T} ! h^{3 N_{T}}\right]$. Then the total entropy of the composite system is just given by the sum of the subsystem entropies. But this choice is not required.

\section{THE APPLICATION OF THE ENTROPY EQUATIONS}

Eqs. (8), (9), and (10) are intended to be applied to the set of all systems in the world that can be regarded as classical. That includes not only systems in a particular laboratory, but also those in a different city or continent. Most systems will not interact with each other because of physical separation, and the constraints of their not exchanging energy, volume, or particles are expected to remain indefinitely.

The entropy of a single system is given by Eq. (10). For experiments involving only a local group of systems (or subsystems of the overall composite system), the existence of many other (sub)systems can be safely ignored, because their properties do not affect the local thermodynamic variables. Similarly, the value of the additive constants in Eq. (8) will not affect the predictions of any experiment.

Eqs. (8) and (10) allow us to find the non-negative change in total entropy $\left(\Delta S_{T} \geq 0\right)$ during any irreversible process between equilibrium states that occurs after the release of a constraint, as well as the final equilibrium values of thermodynamic observables.

Dieks has criticized this derivation of the entropy. I discuss his views in the next section.

\section{DIEKS' OBJECTION}

Dieks' criticism rests on the claim that the choice of additive constant, $X$ in Eq. (8), is essential for obtaining my results for the entropy 11]. This claim is untenable, since I have derived the entropy of an arbitrary subsystem [Eq. (10)] without fixing the value of $X$, and the value of $X$ has no physical consequences.

Looking further, we can see that Dieks means something different. He is interested in the value of the entropy of the entire composite system of $M$ subsystems for the case in which all constraints have been released. As shown above in Eq. (11), the release of all constraints leads to a constant $S_{T} \rightarrow X$, where $X$ is arbitrary. Dieks is concerned about the determination of a particular form of this constant. Since there are no physical consequences for any value of $X$, I fail to see the importance of the issue.

Dieks explicitly recognizes that this issue is without importance. Writing $N$ for what I have called $N_{T}$, he says in a footnote:

A more detailed discussion should also take into account that the division by $N$ ! is without significance anyway as long as $N$ is constant [11].

However, he still uses the value of this constant to frame his objection to my definition. The reason for this contradiction might lie in his incorrect description of my definition of entropy, which he claims amounts simply to dividing the traditional expression by $N$ !.

I will consider his argument in detail.

\section{V.1. Two simple subsystems}

Dieks considered an isolated composite system consisting of only two ideal gases $(M=2)$, and simplified his analysis by ignoring the energy dependence. In discussing his argument, I will depart from Dieks' notation 27] by using $N_{T}=N_{1}+N_{2}$ as the constant total number of particles to be consistent with the notation I used in previous sections. For clarity, I will also retain an arbitrary value of the additive constant $X$ (see Eq. (7), above) until the end of the discussion, although Dieks makes the specific choice of $X=k_{B} \ln \left(V_{T}^{N_{T}} / N_{T}\right.$ ! $)$, "for reasons of convenience," early in his argument [11].

For Dieks' two subsystems of classical ideal gases, my 
Eq. (8) becomes his Eq. (2),

$$
\begin{aligned}
S_{T}\left(N_{1}, V_{1} ; N_{2}, V_{2}\right)= & k_{B} \ln \left(\frac{N_{T} !}{N_{1} ! N_{2} !} \frac{V_{1}^{N_{1}} V_{2}^{N_{2}}}{V_{T}^{N}}\right)+X \\
= & k_{B} \ln \left(\frac{V_{1}^{N_{1}}}{N_{1} !}\right)+k_{B} \ln \left(\frac{V_{2}^{N_{2}}}{N_{2} !}\right) \\
& -k_{B} \ln \left(\frac{V_{T}^{N_{T}}}{N_{T} !}\right)+X,
\end{aligned}
$$

where $N_{T}=N_{1}+N_{2}$ and $V_{T}=V_{1}+V_{2}$ are constants. Note that Dieks' choice for the value of the constant $X$ means that the last two terms in Eq. (12) cancel in his Eq. (2).

Since Eq. (12) is valid for all values of $N_{1}, N_{2}, V_{1}$, and $V_{2}$, we immediately have the (partial) entropies,

$$
S_{j}\left(V_{j}, N_{j}\right)=k_{B} \ln \left(\frac{V_{j}^{N_{j}}}{N_{j} !}\right),
$$

where $j=1$ or 2 . I claim that this is a proper derivation of the factors $1 / N_{j} !$.

Dieks made the following comment on his Eq.(2) (writing $N$ for what I have called $N_{T}$ ).

Indeed, the dependence of the total entropy in Eq. (2) on $N_{1}$ and $N_{2}$ is unrelated to how $N$ occurs in this formula (and to the choice of the zero of the total entropy) [11].

His comment confirms the validity of my derivation of the factors $1 / N_{1}$ ! and $1 / N_{2}$ ! in the entropies of subsystems 1 and 2, as well as the irrelevance of the value of the additive constant $X$.

Dieks then calculates the entropy after the release of the constraint on the particle number and return to equilibrium. He gets the result $X=k_{B} \ln \left(V_{T}^{N_{T}} / N_{T} !\right)$. Dieks claims that this was the way I had obtained a $-k_{B} \ln N_{T}$ ! dependence of the total entropy. I did not fix the value of $X$, so I did not derive an expression for the entropy after the release of constraints.

Actually, the form of the $k_{B} \ln \left(V_{T}^{N_{T}} / N_{T} !\right)$ term in the joint entropy does not come from choosing the constant $X$ to make $S_{T}=\sum_{j=1}^{M} S_{j}$, but rather from the simplicity of the example used. If the properties of the subsystems are generalized, a different result is obtained.

\section{V.2. Two less simple subsystems}

Consider the entropy,

$$
S_{j}=k_{B} N_{j}\left[\frac{3}{2} \ln \left(\frac{E_{j}-N_{j} a_{j}}{N_{j}}\right)+\ln \left(\frac{V_{j}}{N_{j}}\right)+Y_{j}^{\prime}\right],
$$

where I have used Stirling's approximation. The total entropy before allowing the systems to interact is
$S_{T}=S_{1}+S_{2}$. The energy dependence is now given explicitly, and an energy shift per particle, $a_{j}$, is given to each subsystem. Assume that $a_{1}=0$ and $a_{2}>0$. Let subsystems 1 and 2 come into thermal contact and exchange energy and particles.

The temperature dependence of the energy in the $j$-th subsystem is given by

$$
E_{j}=\frac{3}{2} k_{B} N_{j} T_{j}+N_{j} a_{j},
$$

so the condition of equilibrium with respect to energy exchange is

$$
\frac{E_{1}^{\prime}}{N_{1}}=\frac{E_{2}^{\prime}}{N_{2}}-a_{2},
$$

where I have indicated the new values of the energies by $E_{1}^{\prime}$ and $E_{2}^{\prime}$.

Now let the two subsystems exchange particles. From the condition of equilibrium with respect to particle number, it is straightforward to derive

$$
\ln \left(\frac{V_{1}}{N_{1}^{\prime \prime}}\right)=\ln \left(\frac{V_{2}}{N_{2}^{\prime \prime}}\right)-\frac{3}{2}\left[\frac{1}{E_{2}^{\prime \prime} / N_{2}^{\prime \prime} a_{2}-1}\right],
$$

where I have indicated the new values of the energies and particle numbers by double primes, i.e: $E_{j}^{\prime}$ and $N_{j}^{\prime \prime}$. Since $E_{1}^{\prime \prime} / N_{1}^{\prime \prime} \neq E_{2}^{\prime \prime} / N_{2}^{\prime \prime}$ and $V_{1} / N_{1}^{\prime \prime} \neq V_{2} / N_{2}^{\prime \prime}$, the total entropy cannot be written as a function of $\left(E_{1}^{\prime \prime}+E_{2}^{\prime \prime}\right),\left(V_{1}+V_{2}\right)$, and $\left(N_{1}^{\prime \prime}+N_{2}^{\prime \prime}\right)$. There is no term in $S_{T}^{\prime \prime}=S_{1}^{\prime \prime}\left(E_{1}^{\prime \prime}, V_{1}, N_{1}^{\prime \prime}\right)+S_{2}^{\prime \prime}\left(E_{2}^{\prime \prime}, V_{2}, N_{2}^{\prime \prime}\right)$ of the form $k_{B} \ln \left(V_{T}^{N_{T}} / N_{T} !\right)$.

For the next example it will be sufficient to again consider ideal gases and ignore the energy dependence.

\section{V.3. Three simple subsystems}

Dieks' analysis does not recognize that the thermodynamic variables in subsystems 1 and 2 remain $N_{1}$ and $N_{2}$, even after the systems come to equilibrium. They are not replaced by a single variable. This can be seen most easily by considering $M \geq 3$ subsystems. To avoid confusion, denote the number of particles in subsystems 1 and 2 by $N_{1,2}=N_{1}+N_{2}$, because it is no longer constant. Now consider how subsystems 1 and 2 interact with a third subsystem.

Let subsystems 1 and 2 first come to equilibrium and then be separated again, denoting the new particle numbers by $N_{1}^{\prime}$ and $N_{2}^{\prime}$. Let subsystem 3 originally have a high number density, $N_{3} / V_{3}>N_{1}^{\prime} / V_{1}=N_{2}^{\prime} / V_{2}$. Now let subsystem 2 exchange particles with system 3 , so that $N_{2}$ increases $\left(N_{2}^{\prime \prime}>N_{2}^{\prime}\right)$. Subsystems 2 and 3 come to a new equilibrium, for which

$$
\frac{N_{1}^{\prime}}{V_{1}}<\frac{N_{2}^{\prime \prime}}{V_{2}}=\frac{N_{3}^{\prime \prime}}{V_{3}}
$$


The entropy of subsystems 1 and 2 is (with Stirling's approximation),

$$
S_{1}^{\prime}+S_{2}^{\prime \prime} \approx k_{B} N_{1}^{\prime} \ln \left(\frac{V_{1}}{N_{1}^{\prime}}\right)+k_{B} N_{2}^{\prime \prime} \ln \left(\frac{V_{2}}{N_{2}^{\prime \prime}}\right) .
$$

Since the number density is different in subsystems 1 and 2 , it is clear that $S_{1}^{\prime}+S_{2}^{\prime \prime}$ is not given by $k_{B} N_{1,2} \ln \left(V_{1,2} / N_{1,2}\right)$.

\section{V.4. An arbitrary number of subsystems}

When Dieks discusses the case of many systems, he writes that I require a "consistency" condition,

that the entropy formula should be such that there will be no change in entropy when a partition is removed[11].

I do not require it, and it is not a consistency condition. It is the condition that systems separated by a partition are in equilibrium, which is not generally true in the presence of a constraint.

To summarize, I have calculated the dependence of the entropy on the variables $\left\{E_{j}, V_{j}, N_{j} \mid=1, \ldots, M\right\}$ in the presence or absence of arbitrary constraints. My definition enables the calculation of the equilibrium conditions and entropy changes. The additive constant, $X$, may be determined by convention.

\section{PETERS' OBJECTION}

A prominent question in the literature is whether entropy should be defined in one step or two. The two-step approach can be described as hybrid because it starts with a definition of entropy, notes that the definition fails in some respect, and then corrects it to agree more closely with the thermodynamic properties of entropy. The historical reason for this peculiar question lies in the effort to maintain a definition of entropy in the form of the logarithm of a volume in phase space by modifying it to correct the dependence on particle number 11 13, 2830]. Since this process usually involves the inclusion of a negative term, $-k_{B} \ln N$ !, the result is often called a "reduced entropy."

Peters has introduced an interesting hybrid definition of the entropy 13,14 . In doing so, he explicitly rejected the derivation of entropy given in Section III although his only criticism turns out to be something we agree on. We both recognized that macroscopic experiments do not identify individual particles, so we can never know which particles are in which system. However, Peters claimed that my version was "imprecise" because it did not include the condition he denoted as being "harmonic," defined as follows.

Systems for which all possible particle compositions are equiprobable will be called harmonic 13.
For comparison, I had written that,

when a system of distinguishable particles is allowed to exchange particles with the rest of the world, we must include the permutations of all possible combinations of particles that might enter or leave the system [6].

It is clear that we have made essentially the same assumption.

Peters' takes a hybrid approach in that he chooses to define a form of the Shannon entropy, and then "reduces" it to arrive at the final form [13, 31].

$$
\begin{aligned}
R_{P}= & -k_{B} \sum_{i=1}^{M} \int d^{3 N_{i}} p_{i} \int d^{3 N_{i}} q_{i} \\
& \times \rho_{i}\left(p_{i}, q_{i}\right) \ln \left(\rho_{i}\left(p_{i}, q_{i}\right) h^{3 N_{i}}\right) \\
& -k_{B} \ln N !
\end{aligned}
$$

This form does have the correct $N$-dependence, and for the correct reason. However, $R_{P}$ fails to satisfy the second law of thermodynamics.

In Section 4.3.3.2 of Ref. [13], Peters discusses an irreversible process initiated by the release of constraints to allow exchange of energy and particles between two subsystems. He assumes that "both before and after the exchange" the two subsystems "are in microcanonical equilibrium." The problem is that this assumption is contradicted by Liouville's theorem, which requires the total time derivative of the probability distribution in the phase space of the complete composite system to vanish. This means $R_{P}$ does not increase during an irreversible process, so it does not satisfy the second law of thermodynamics.

Peters explicitly acknowledges the difficulty posed by Liouville's theorem in his Section 5.6.5, writing that, "the Liouville equation is entropy conserving and therefore cannot describe irreversible processes." He does not comment on the contradiction between his Sections 4.3.3.2 and 5.6.5.

In contrast, the Liouville equation does not conserve the entropy as defined in this paper, and the Second Law is satisfied.

\section{SUMMARY}

I've argued for a definition of the thermodynamic entropy based on the probability distribution of the macroscopic variables in a composite system. The entropy defined this way satisfies the postulates for thermodynamics [1 $[3]$. I've addressed the objections by Dieks [11, 12] and Peters [13, 14] to this derivation of the entropy from statistical mechanics and shown that they are not valid.

Since the thermodynamic entropy is known to be unique apart from constants chosen by convention [4], any other valid definition of the entropy must be equivalent the one presented here. 


\section{ACKNOWLEDGEMENT}

I would like to thank Roberta Klatzky for many helpful discussions. This research did not receive any specific grant from funding agencies in the public, commercial, or not-for-profit sectors.
1. L. Tisza, Generalized Thermodynamics (MIT Press, Cambridge, 1966).

2. H. B. Callen, Thermodynamics and an Introduction to Thermostatistics, 2nd ed. (Wiley, New York, 1985).

3. R. H. Swendsen, An Introduction to Statistical Mechanics and Thermodynamics (Oxford, London, 2012).

4. E. H. Lieb and J. Yngvason, "The physics and mathematics of the second law of thermodynamics," Physics Reports, 310, 1-96 (1999).

5. R. H. Swendsen, "Statistical mechanics of classical systems with distinguishable particles," J. Stat. Phys., 107, 1143-1165 (2002).

6. R. H. Swendsen, "Statistical mechanics of colloids and Boltzmann's definition of the entropy," Am. J. Phys., 74, 187-190 (2006).

7. R. H. Swendsen, "Gibbs' paradox and the definition of entropy," Entropy, 10, 15-18 (2008).

8. R. H. Swendsen, "How physicists disagree on the meaning of entropy," Am. J. Phys., 79, 342-348 (2011).

9. R. H. Swendsen, "Choosing a definition of entropy that works," Found. of Physics, 42, 582-593 (2012).

10. R. H. Swendsen, "Unnormalized probability: A different view of statistical mechanics," Am. J. Phys., 82, 941-946 (2014).

11. D. Dieks, "Is there a unique physical entropy? micro versus macro," in New Challenges to Philosophy of Science (The Philosophy of Science in a European Perspective), edited by H. Andersen, D. Dieks, W. Gonzalez, T. Uebel, and G. Wheeler (Springer, New York, 2013) pp. 23-34.

12. D. Dieks, "The logic of identity: Distinguishability and indistinguishability in classical and quantum physics," Foundations of Physics, 44, 1302-1316 (2014).

13. H. Peters, "Statistics of distinguishable particles and resolution of the Gibbs paradox of the first kind," J. Stat. Phys., 141, 785-828 (2010).

14. H. Peters, "Demonstration and resolution of the Gibbs paradox of the first kind," Eur. J. Phys., 35, 015023 (2014).

15. I will only write the equations for a single type of particle. The generalization to several different kinds of particles is trivial, but requires indices that might obscure the essential argument.

16. R. H. Swendsen, "Continuity of the entropy of macroscopic quantum systems," Phys. Rev. E, 92, 052110 (2015).

17. R. H. Swendsen, "The ambiguity of 'distinguishability' in statistical mechanics," Am. J. Phys., 83, 545-554 (2015).

18. R. H. Swendsen, "Explaining irreversibility," Am. J. Phys., 76, 643-648 (2008).

19. O. Penrose, Foundations of Statistical Mechanics: A Deductive Treatment (Pergamon, Oxford, 1970).

20. S. Goldstein and J. L. Lebowitz, "On the (Boltzmann) entropy of nonequilibrium systems," Physica D: Nonlinear Phenomena, 193, 5366 (2004).
21. J. L. Lebowitz, "From time-symmetric microscopic dynamics to time-asymmetric macroscopic behavior: An overview," in Boltzmann's Legacy, edited by G. Gallavotti, W.L. Reiter, and J. Yngvason (European Mathematical Society, Zurich, 2007) pp. 63-88.

22. R. H. Swendsen and J.-S. Wang, "The Gibbs volume entropy is incorrect," Phys. Rev. E., 92, 020103(R) (2015).

23. L. Boltzmann, "Über die Beziehung zwischen dem zweiten Hauptsatze der mechanischen Wärmetheorie und der Wahrscheinlichkeitsrechnung respektive den Sätzen über das Wärmegleichgewicht," Wien. Ber., 76, 373-435 (1877), reprinted in Wissenschaftliche Abhandlungen von Ludwig Boltzmann (Chelsea, New York Vol. II, pp. 164-223.

24. K. Sharp and F. Matschinsky, "Translation of Ludwig Boltzmann's paper on the relationship between the second fundamental theorem of the mechanical theory of heat and probability calculations regarding the conditions for thermal equilibrium, Sitzungberichte der Kaiserlichen Akademie der Wissenschaften. Mathematisch-Naturwissen Classe. abt. ii, lxxvi 1877, pp 373-435 (Wien. Ber. 1877, 76:373-435). reprinted in Wiss. Abhandlungen, vol. ii, reprint 42, p. 164-223, Barth, Leipzig, 1909," Entropy, 17, 1971-2009 (2015), ISSN 1099-4300.

25. M. Planck, "Über das Gesetz der Energieverteilung im Normalspektrum," Drudes Annalen, 553, 65-74 (1901), reprinted in Ostwalds Klassiker der exakten Wissenschaften, Band 206, "Die Ableitung der Strahlungsgesteze".

26. M. Planck, Theorie der Wärmestrahlung (J. A. Barth, Leipzig, 1906) translated into English by Morton Masius in M. Planck, The Theory of Heat Radiation, (Dover, New York, 1991).

27. Readers interested in following the original phrasing of the argument in Dieks' paper may encounter some difficulties caused by the notation [11]. The paper begins by using the symbol $N$ to denote the number of particles in a simple system. When a composite system is first discussed in connection with Dieks' Eq.(1), the particle numbers for the two simple subsystems are $N_{1}$ and $N_{2}$, while $N=N_{1}+N_{2}$ has become a constant that denotes the total number of particles in the isolated composite system. In his Eq.(3), $N$ is back to being a variable that denotes the particle number in a simple system. In his Eq.(4), $N$ is again the total number of particles in the isolated composite system, and again a constant.

28. D. Dieks and M. A. M. Versteegh, "Identical quantum particles and weak discernibility," Found. Phys., 38, 923-934 (2008).

29. M. A. M. Versteegh and D. Dieks, "The Gibbs paradox and the distinguishability of identical particles," Am. J.Phys., 79, 741-746 (2011).

30. C. H. Cheng, "Thermodynamics of the system of distinguishable particles," Entropy, 11, 326-33 (2009). 
31. C. E. Shannon, "A mathematical theory of communication," Bell System Technical Journal, 27, 623-656, 379423 (1948). 